\title{
HD 16771: A lithium-rich giant in the red-clump stage
}

\author{
Arumalla B. S. Reddy and David L. Lambert
}

\begin{abstract}
W.J. McDonald Observatory and Department of Astronomy, The University of Texas at Austin, Austin, TX 78712-1205, USA e-mail: bala@astro.as.utexas.edu
\end{abstract}

Received 16 February 2016 / Accepted 15 March 2016

\begin{abstract}
Aims. We report the discovery of a young lithium rich giant, HD 16771, in the core-helium burning phase that does not seem to fit existing proposals of $\mathrm{Li}$ synthesis near the luminosity function bump or during He-core flash. We aim to understand the nature of $\mathrm{Li}$ enrichment in the atmosphere of HD 16771 by exploring various Li enhancement scenarios.

Methods. We have collected high-resolution echelle spectra of HD 16771 and derived stellar parameters and chemical abundances for 27 elements by either line equivalent widths or synthetic spectrum analyses.

Results. HD 16771 is a Li-rich $(\log \epsilon(\mathrm{Li})=+2.67 \pm 0.10 \mathrm{dex})$ intermediate mass giant star $\left(M=2.4 \pm 0.1 M_{\odot}\right)$ with age $\sim 0.76 \pm$ $0.13 \mathrm{Gyr}$ and located at the red giant clump. Kinematics and chemical compositions are consistent with HD 16771 being a member of the Galactic thin disk population. The non-detection of ${ }^{6} \mathrm{Li}(<3 \%)$, a low carbon isotopic ratio $\left({ }^{12} \mathrm{C} /{ }^{13} \mathrm{C}=12 \pm 2\right)$, and the slow rotation $\left(v \sin i=2.8 \mathrm{~km} \mathrm{~s}^{-1}\right)$ all suggest that lithium might have been synthesized in this star. On the contrary, HD 16771 with a mass of $2.4 M_{\odot}$ has no chance of encountering luminosity function bump and He-core flash where the possibility of fast deep-mixing for $\mathrm{Li}$ enrichment in $\mathrm{K}$ giants has been suggested previously.

Conclusions. Based of the evolutionary status of this star, we discuss the possibility that ${ }^{7} \mathrm{Li}$ synthesis in HD 16771 is triggered by the engulfment of close-in planet(s) during the RGB phase.
\end{abstract}

Key words. stars: fundamental parameters - stars: abundances - stars: individual: HD 16771 - stars: late-type - stars: atmospheres techniques: spectroscopic

\section{Introduction}

Lithium depletion in giants is a natural consequence of stellar evolution. Soon after stars leave the main sequence the outer layers expand and cool, a star's rotation rate slows, the convection zone deepens and sweeps to the surface material from internal layers. This first dredge-up causes an overall dilution of $\mathrm{Li}$ abundance by about 1-2 dex (Iben 1967a,b). Indeed, abundance analysis of $\mathrm{G}$ and $\mathrm{K}$ giants (Brown et al. 1989) even show much lower $\mathrm{Li}$ abundances in many giants than the expectations of standard stellar models (Iben 1967a,b) indicative of significant Li depletion during the pre-main and/or main sequence phases, and to some extent due to non-canonical mixing on the red giant branch (RGB). At the end of first dredger-up, significant abundance alterations to the giant's atmosphere are the depletion of fragile $\mathrm{Li}$, increase in ${ }^{4} \mathrm{He},{ }^{14} \mathrm{~N},{ }^{13} \mathrm{C}$, and dilution of ${ }^{12} \mathrm{C}$ thereby lowering the ${ }^{12} \mathrm{C} /{ }^{13} \mathrm{C}$ ratio down to $\sim 20-30$ from its initial value of 90 on the main sequence (Charbonnel et al. 1998).

In spite of this convective mixing, a fraction of giants in the Galactic disk exhibit abnormally high Li abundances exceeding by nearly 2-3 orders of magnitude the standard model predictions for stars on the $\mathrm{RGB}^{1}$ (Brown et al. 1989; Charbonnel \& Balachandran 2000; Kumar et al. 2011), and a few of them present rapid rotation (see Carlberg 2012 and references therein), strong infrared (IR) excesses (de La Reza 1996), and anomalously low ${ }^{12} \mathrm{C} /{ }^{13} \mathrm{C}$ ratios (Sneden 1986). Proposals that giants synthesize ${ }^{7} \mathrm{Li}$ via Cameron-Fowler (Cameron \& Fowler 1971) ${ }^{7}$ Be-transport mechanism near RGB

\footnotetext{
$1 \mathrm{~K}$ giants with $\log \epsilon(\mathrm{Li})>1.5 \mathrm{dex}$ are treated as $\mathrm{Li}$-rich while those with Li abundances exceeding the present interstellar medium abundance, $\log \epsilon(\mathrm{Li})>3.1 \mathrm{dex}$, are termed as super Li-rich.
}

bump in low mass stars (Charbonnel \& Balachandran 2000) and/or during the He-core flash in stars of $M<2.2 M_{\odot}$ (Kumar et al. 2011) have been challenged by the discovery of super Li-rich giants at magnitudes that span the RGB (Monaco et al. 2011; Martell \& Shetrone 2013). These observations imply that Li enrichment may not be limited to specific stellar evolutionary stages. Numerous models proposed hitherto are far from reaching a consensus on the synthesis of $\mathrm{Li}$ in $\mathrm{K}$ giants which may partly be attributed to the paucity of data on Li-rich K giants (see e.g. Monaco et al. 2011; and Carlberg et al. 2012, and references therein).

Here we report the detection of a warm Li-rich clumpgiant, a few of them found previously by Brown et al. (1989) and Martell \& Shetrone (2013), whose mass and location in the Hertzsprung-Russell (HR) diagram is incompatible with an encounter with the luminosity function bump (LFB) or $\mathrm{He}$-core flash where the internal production of $\mathrm{Li}$ in giants was proposed previously (Charbonnel \& Balachandran 2000; Kumar et al. 2011). Here, we restrict ourselves to discussing the chemical composition of HD 16771 and its evolutionary state in the HR diagram with an emphasis on providing a plausible scenario for the observed Li enrichment.

\section{Observations and data reduction}

The sample red giant star HD 16771 analysed in this letter was selected as a part of the program dedicated to measure chemical abundances for red giants in open clusters (OCs; Reddy et al. 2012, 2013, 2015). High-resolution $(R \sim 60000)$ and high signal-to-noise ratio (S/N) optical spectra of HD 16771 were acquired during the night of 2013 November with the 
Robert G. Tull coudé cross-dispersed echelle spectrograph (Tull et al. 1995) at the 2.7-m Harlan J. Smith reflector of the McDonald observatory. The spectra were reduced to 1D in multiple steps using various routines available in the standard spectral reduction software IRAF $^{2}$.

The wavelength range 3600-9800 A spread across various echelle orders covered in a single $1200 \mathrm{~s}$ exposure is sufficient to perform an abundance analysis of many elements. The spectrum has a $\mathrm{S} / \mathrm{N}$ of about 220 as measured around $6000 \AA$ region and about 250 near the lithium resonance doublet at $6707.8 \AA$, while at wavelengths shorter than $4000 \AA$ the $\mathrm{S} / \mathrm{N}$ decreases and falls to 35 around $3600 \AA$ region. We measured a heliocentric radial velocity (RV) of $+6.9 \pm 0.1 \mathrm{~km} \mathrm{~s}^{-1}$ which is in fair agreement with Mermilliod et al. (2008) value of $+7.1 \pm 0.3 \mathrm{~km} \mathrm{~s}^{-1}$. The methods of data reduction and RV measurements are described in detail in Reddy et al. (2012, 2013, 2015).

Although HD 16771 as mentioned in the SIMBAD ${ }^{3}$ astronomical database is a member of the OC NGC 1039/M 34, both the proper motions $\left(\mu_{\alpha} \cos \delta, \mu_{\delta}\right)$ and RV measurements of the star differ by $(+38,-11)$ mas/yr and $16 \mathrm{~km} \mathrm{~s}^{-1}$ from those values measured for the cluster dwarfs (Jones \& Prosser 1996). Hence, we consider the star as a member of the Galactic disk in the cluster field.

\section{Abundance analysis}

We performed a differential abundance analysis relative to the Sun by running the abfind driver of Moog (Sneden 1973) adopting the 1D model atmospheres (Castelli \& Kurucz 2004) and the iron line equivalent widths (EWs) following our local thermodynamic equilibrium (LTE) abundance analysis technique described in Reddy et al. (2012, 2013, 2015). The atmospheric parameters are derived by force-fitting the model generated iron line EWs to the observed ones by imposing the excitation and ionization equilibrium and the independence between the iron abundances and EWs. The final stellar parameters of HD 16771 derived using the iron lines are: effective temperature $\left(T_{\text {eff }}\right)=$ $5050 \pm 50 \mathrm{~K}$, surface gravity $(\log g)=2.7 \pm 0.1 \mathrm{~cm} \mathrm{~s}^{-2}$, microturbulence $\left(\xi_{t}\right)=1.35 \pm 0.08 \mathrm{~km} \mathrm{~s}^{-1}$ and the iron abundance $[\mathrm{Fe} / \mathrm{H}]=-0.12 \pm 0.05$ dex.

In most cases, the abundances are derived from the measured EWs but a few lines were analysed with synthetic spectra. We used a standard synthetic profile fitting procedure (Reddy et al. 2015) and smoothed the computed profiles before matching to the observed ones with Gaussian profiles representing the instrumental profile, and then macroturbulence $\left(V_{\text {macro }}\right)$ and the rotation $(v \sin i)$ profile of the star. The instrumental broadening was measured as the average of widths of three thorium-argon lines in the same echelle order as the stellar feature analysed by spectrum synthesis. We derived an average $v \sin i$ of $2.8 \pm 0.6 \mathrm{~km} \mathrm{~s}^{-1}$ and $V_{\text {macro }}=3.0 \pm 0.4 \mathrm{~km} \mathrm{~s}^{-1}$ by fitting relatively isolated iron lines at 5307.4, 5141.7, 5466.9, 6187.9, 6705.1, 6739.5 and $6750.1 \AA$ following the procedure described in Carlberg et al. (2012).

\footnotetext{
2 IRAF is a general purpose software system for the reduction and analysis of astronomical data distributed by NOAO, which is operated by the Association of Universities for Research in Astronomy, Inc. under cooperative agreement with the National Science Foundation.

3 http://simbad.u-strasbg.fr/simbad/
}

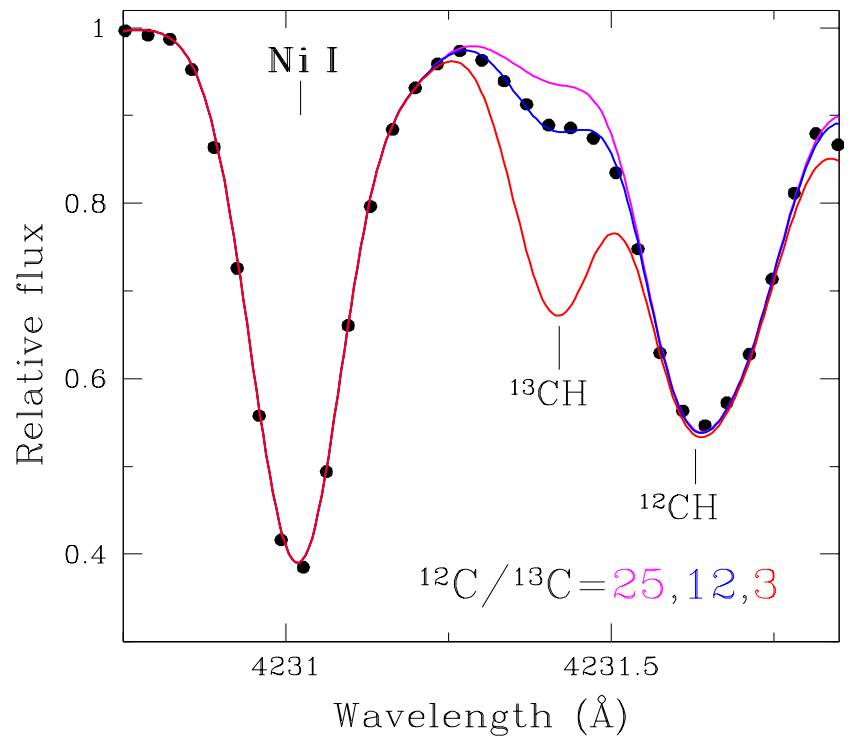

Fig. 1. Synthetic spectra fit to the observed ${ }^{12} \mathrm{CH}$ and ${ }^{13} \mathrm{CH}$ features of HD 16771 around the 4231 A region for a range of ${ }^{12} \mathrm{C} /{ }^{13} \mathrm{C}$ ratios.

\subsection{Li, CNO abundances and ${ }^{12} \mathrm{C}^{/ 3} \mathrm{C}$ ratios}

The abundances of lithium, carbon, nitrogen, oxygen as well as the carbon ${ }^{12} \mathrm{C} /{ }^{13} \mathrm{C}$ isotopic ratios were derived by fitting synthetic spectra to selected regions of observed spectrum. As the $\mathrm{Li}$ resonance doublet at $6707.8 \AA$ is blended with several weak $\mathrm{CN}$, $\mathrm{Si}, \mathrm{Ca}, \mathrm{V}$ and Fe features (Ghezzi et al. 2009), it is necessary to predetermine their abundances before analysing the lithium feature.

We obtained a carbon abundance of $[\mathrm{C} / \mathrm{H}]=-0.32 \pm 0.06$ from the analysis of $\mathrm{C}_{2}$ Swan $(1,0)$ lines at $5135 \AA$ and the permitted carbon line at $5380 \AA$. The nitrogen abundance of $[\mathrm{N} / \mathrm{H}]=$ $+0.25 \pm 0.06$ was derived by modelling of several ${ }^{12} \mathrm{C}^{14} \mathrm{~N}$ lines in the $6332 \AA$ and $7938 \AA$ regions and, the oxygen abundance of $[\mathrm{O} / \mathrm{H}]=-0.08 \pm 0.05$ from the analysis of IR triplet $(7772$, 7774 and $7775 \AA$ ) and the forbidden line at $6300 \AA$. The oxygen abundance derived from the IR triplet was adjusted for the nonLTE effects by adopting empirical non-LTE corrections from Afşar et al. (2012). Here, we referred the $\mathrm{C}, \mathrm{N}$ and $\mathrm{O}$ abundances of HD 16771 to the solar abundances of $\log \epsilon(\mathrm{C})=8.37 \mathrm{dex}$, $\log \epsilon(\mathrm{N})=7.98 \mathrm{dex}$, and $\log \epsilon(\mathrm{O})=8.83$ dex derived using our linelists.

The carbon ${ }^{12} \mathrm{C} /{ }^{13} \mathrm{C}$ isotopic ratio, which is an informative diagnostic of mixing processes (Charbonnel et al. 1998) operating in the stellar atmospheres during the evolution of red giants, was measured from the synthesis of ${ }^{12} \mathrm{CH}$ and ${ }^{13} \mathrm{CH}$ features (Lambert \& Dearborn 1972) at $4231 \AA$ (Fig. 1). We derived a ${ }^{12} \mathrm{C} /{ }^{13} \mathrm{C}$ ratio of $12 \pm 2$, indicating that the dredge-up episodes have thoroughly mixed the surface material with the stellar interior. The molecular data for $\mathrm{C}_{2}$ comes from Ram et al. (2014), $\mathrm{CH}$ and $\mathrm{CN}$ data are taken from Masseron et al. (2014) and Sneden et al. (2014), respectively.

Thereafter, we measured the lithium abundance from the synthesis of resonance doublet at $6707.8 \AA$ adopting the hyperfine structure data from Ghezzi et al. (2009). A fit to the observed lithium profile without the need for ${ }^{6} \mathrm{Li}$, as shown in Fig. 2, is obtained for a LTE abundance of $\log \epsilon(\mathrm{Li})=+2.67 \pm 0.10 \mathrm{dex}$. Our LTE lithium abundance was corrected for the non-LTE corrections provided by Lind et al. (2009) for a star of given $T_{\text {eff }}$, $\log g, \xi_{t}$ and $[\mathrm{Fe} / \mathrm{H}]$. 


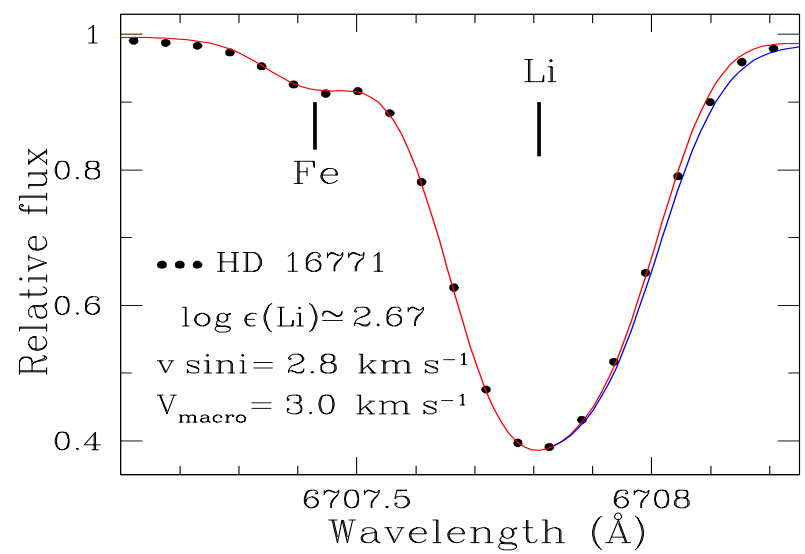

Fig. 2. Best synthetic profile fit (red) to the observed Li $6707 \AA$ line in the spectra of HD 16771. The vertical lines represent the central wavelengths of those species. Also shown is the spectrum (blue) computed for $3 \%$ of ${ }^{6} \mathrm{Li}$.

As HD 16771 has no measured stellar parallax $(\pi)$, initial guess of $\pi$ was made by incorporating the derived spectroscopic atmospheric parameters into the known relation

$$
\begin{aligned}
\log \pi= & 0.5\left[\log \left(g / g_{\odot}\right)-\log \left(M / M_{\odot}\right)-4 \log \left(T_{\text {eff }} / T_{\text {eff }} \odot\right)\right] \\
& -0.2\left(V-A_{V}+B C_{V}+0.25\right)
\end{aligned}
$$

where $A_{V}$ is the interstellar extinction in $V$ band and the bolometric correction $B C_{V}$ is estimated from the spectroscopic $T_{\text {eff }}$ and $[\mathrm{Fe} / \mathrm{H}]$ using Alonso et al. (1999) calibration. Here we adopt $\log g_{\odot}=4.44 \mathrm{~cm} \mathrm{~s}^{-2}$ and $T_{\text {eff }, \odot}=5777 \mathrm{~K}$ and assume that HD 16771 has a mass of $M=2.0 M_{\odot}$.

Using the Johnson $V$ magnitude along with the spectroscopic $T_{\text {eff }}$ and $[\mathrm{Fe} / \mathrm{H}]$, our guess of $\pi=4.6$ mas is varied until the surface gravity estimate from the PARAM ${ }^{4}$ code (da Silva et al. 2006) and PARSEC stellar evolutionary tracks (Bressan et al. 2012) matches our spectroscopically determined value of $\log g$, i.e., $2.7 \mathrm{~cm} \mathrm{~s}^{-2}$. We adopted the stellar mass and age of HD 16771 from the isochrone that corresponds to our best measured value of $\pi=3.95 \pm 0.15$ mas.

The spatial velocities $\left(U_{\mathrm{LSR}}, V_{\mathrm{LSR}}, W_{\mathrm{LSR}}\right)=(-24.9,-29.8$, 10.0) $\mathrm{km} \mathrm{s}^{-1}$, distance away from the Galactic plane $z=$ $0.07 \mathrm{kpc}$, and a thin disk membership probability (Reddy et al. 2015) of $98 \%$ along with the chemical composition (see Table 1) of HD 16771 agree well for an object typical from the Galactic thin disk. A summary of stellar parameters of HD 16771 is presented in Table 2.

\section{Discussion}

According to the PARSEC evolutionary tracks (Bressan et al. 2012) and a Bayesian estimation method (da Silva et al. 2006), HD 16771 at a distance of $253 \pm 10 \mathrm{pc}$ from the Sun (see Table 2), and with a mass $M=2.4 \pm 0.1 M_{\odot}$ and age $=0.76 \pm$ 0.13 Gyr shares the region enjoyed by the He-core burning clump giants that have evolved to the clump via the RGB tip in the HR-diagram. The position of HD 16771 (large red dot) and the other Li-rich giants extracted from Adamów et al. (2014, 2015, green), Silva Aguirre et al. (2014, magenta), Jofré et al. (2015, purple), and from Kumar et al. (2011, blue) and additional stars collected by Kumar et al. from the literature (black squares) are shown in the HR-diagram (Fig. 3) along with the PARSEC evolutionary tracks computed for stars in the mass range from 0.9 to $3.8 M_{\odot}$ from Bressan et al. (2012).

\footnotetext{
4 http://stev.oapd.inaf.it/cgi-bin/param_1.3
}

\begin{tabular}{|c|c|}
\hline Species & HD 16771 \\
\hline $\log \epsilon(\mathrm{Li})_{\mathrm{LTE}}$ & $+2.67 \pm 0.10(1)$ \\
\hline $\log \epsilon(\mathrm{Li})_{\text {non-LTE }}$ & $+2.64 \pm 0.10$ \\
\hline$[\mathrm{C} \mathrm{I} / \mathrm{Fe}]$ & $-0.20 \pm 0.06$ \\
\hline$[\mathrm{N}$ i/Fe] & $+0.37 \pm 0.06$ \\
\hline [O I/Fe] & $+0.04 \pm 0.05(5)$ \\
\hline$[\mathrm{Na} \mathrm{I} / \mathrm{Fe}]$ & $+0.08 \pm 0.04(5)$ \\
\hline$[\mathrm{Mg} \mathrm{I} / \mathrm{Fe}]$ & $+0.02 \pm 0.04(6)$ \\
\hline$[\mathrm{Al}$ I/Fe] & $-0.04 \pm 0.02(5)$ \\
\hline$[\mathrm{Si} \mathrm{I} / \mathrm{Fe}]$ & $+0.06 \pm 0.03(15)$ \\
\hline$[\mathrm{Ca}$ I/Fe] & $+0.04 \pm 0.05(12)$ \\
\hline$[\mathrm{Sc}$ II $/ \mathrm{Fe}]$ & $+0.10 \pm 0.04(2)$ \\
\hline [Ti I/Fe] & $+0.01 \pm 0.05(16)$ \\
\hline [Ti II/Fe] & $-0.05 \pm 0.04(7)$ \\
\hline [V II $/ \mathrm{Fe}]$ & $+0.05 \pm 0.07(14)$ \\
\hline$[\mathrm{Cr} \mathrm{I} / \mathrm{Fe}]$ & $+0.02 \pm 0.04(10)$ \\
\hline$[\mathrm{Cr}$ II/Fe] & $+0.04 \pm 0.05(6)$ \\
\hline$[\mathrm{Mn} \mathrm{I} / \mathrm{Fe}]$ & $-0.08 \pm 0.07(2)$ \\
\hline$[\mathrm{Fe} \mathrm{I} / \mathrm{H}]$ & $-0.12 \pm 0.05(163)$ \\
\hline$[\mathrm{Fe} \mathrm{II} / \mathrm{H}]$ & $-0.13 \pm 0.05(15)$ \\
\hline$[\mathrm{Co}$ II $/ \mathrm{Fe}]$ & $+0.05 \pm 0.04(6)$ \\
\hline$[\mathrm{Ni} / \mathrm{Fe}]$ & $-0.08 \pm 0.04(27)$ \\
\hline$[\mathrm{Cu}$ II $/ \mathrm{Fe}]$ & $-0.13 \pm 0.04(1)$ \\
\hline [Zn i/Fe] & $-0.03 \pm 0.07(1)$ \\
\hline [Y II/Fe] & $+0.05 \pm 0.05(5)$ \\
\hline [Zr I/Fe] & $+0.17 \pm 0.06(4)$ \\
\hline$[\mathrm{Ba}$ II $/ \mathrm{Fe}]$ & $+0.27 \pm 0.08(1)$ \\
\hline$[\mathrm{La}$ II/Fe] & $+0.08 \pm 0.04(4)$ \\
\hline$[\mathrm{Ce}$ II/Fe] & $+0.12 \pm 0.04(4)$ \\
\hline$[\mathrm{Nd} \mathrm{II} / \mathrm{Fe}]$ & $+0.23 \pm 0.05(13)$ \\
\hline$[\mathrm{sm} \mathrm{II} / \mathrm{Fe}]$ & $+0.23 \pm 0.05(7)$ \\
\hline$[\mathrm{Eu} \mathrm{II} / \mathrm{Fe}]$ & $+0.17 \pm 0.05(1)$ \\
\hline
\end{tabular}

Table 1. Chemical abundances of HD 16771 for elements from Li to Eu.

Notes. The abundances measured by synthesis are presented in bold typeface while the remaining elemental abundances were calculated using the line EWs. Numbers in the parentheses indicate the number of lines used in calculating the abundance of that element. Owing to the grid boundaries, non-LTE corrections for LTE lithium abundance were applied assuming $T_{\text {eff }}=5000, \log g=3.0, \xi_{t}=2.0$ and $[\mathrm{Fe} / \mathrm{H}]=0.0$.

The lithium content of $\log \epsilon\left(\mathrm{Li}_{\text {non-LTE }}\right)=+2.64 \mathrm{dex}$ in the atmosphere of HD 16771 declares the star to be a Li-rich giant but can not be readily explained with $\mathrm{Li}$ synthesis occurring during the LFB or at the He-core flash. HD 16771 with a mass $M=2.4 \pm 0.1 M_{\odot}$ has no chance of encountering the LFB as the RGB finishes (Sackmann \& Boothroyd 1999) before the H-burning shell reaches the $\mu$-barrier and thus no extra-mixing process is expected to trigger $\mathrm{Li}$ production via cool bottom process (Sackmann \& Boothroyd 1999). Also it should not have experienced He-core flash at the RGB tip as the star with mass $M \geq 2.2 M_{\odot}$ starts burning He in its core (Charbonnel \& Lagarde 2010) while on the RGB. Moreover, the star can not have operated the hot bottom burning mechanism (Sackmann \& Boothroyd 1992) probably responsible for Li production in stars high on the Asymptotic Giant Branch (AGB). Even allowing for errors in the ingredients used in the estimation of stellar mass, the location of HD 16771 on the HR diagram supports these conclusions.

In this vein, we note the detection of warm Li-rich clump-giants with masses above $2.2 M_{\odot}$ in two different studies: Martell \& Shetrone (2013) have identified a sample of clump-giants with the combination of $T_{\mathrm{eff}}=5000-5200$, 
Table 2. Summary of the stellar parameters of HD 16771.

\begin{tabular}{lcc}
\hline \multicolumn{1}{c}{ Parameter } & Value & Reference \\
\hline$V(\mathrm{mag})$ & 7.34 & Nicolet $(1978)$ \\
$B-V(\mathrm{mag})$ & 0.94 & Nicolet $(1978)$ \\
parallax (mas) & $3.95 \pm 0.15$ & This work \\
distance $(\mathrm{pc})$ & $253 \pm 10$ & This work \\
$\mathrm{RV}_{\text {helio }}\left(\mathrm{km} \mathrm{s}^{-1}\right)$ & $+6.91 \pm 0.13$ & This work \\
$T_{\text {eff }}(\mathrm{K})$ & $5050 \pm 50$ & This work \\
$\log g\left(\mathrm{~cm} \mathrm{~s}^{-2}\right)$ & $2.7 \pm 0.1$ & This work \\
$\xi_{t}\left(\mathrm{~km} \mathrm{~s}^{-1}\right)$ & $1.35 \pm 0.08$ & This work \\
{$[\mathrm{Fe} / \mathrm{H}](\mathrm{dex})$} & $-0.12 \pm 0.05$ & This work \\
$v \sin i\left(\mathrm{~km} \mathrm{~s}{ }^{-1}\right)$ & $2.8 \pm 0.6$ & This work \\
\hline $\mathrm{Age}(\mathrm{Gyr})$ & $0.76 \pm 0.13$ & This work \\
$M / M_{\odot}$ & $2.4 \pm 0.1$ & This work \\
$R / R_{\odot}$ & $11.1 \pm 0.5$ & This work \\
$\log \left(L / L_{\odot}\right)$ & $1.92 \pm 0.09$ & This work \\
\hline
\end{tabular}

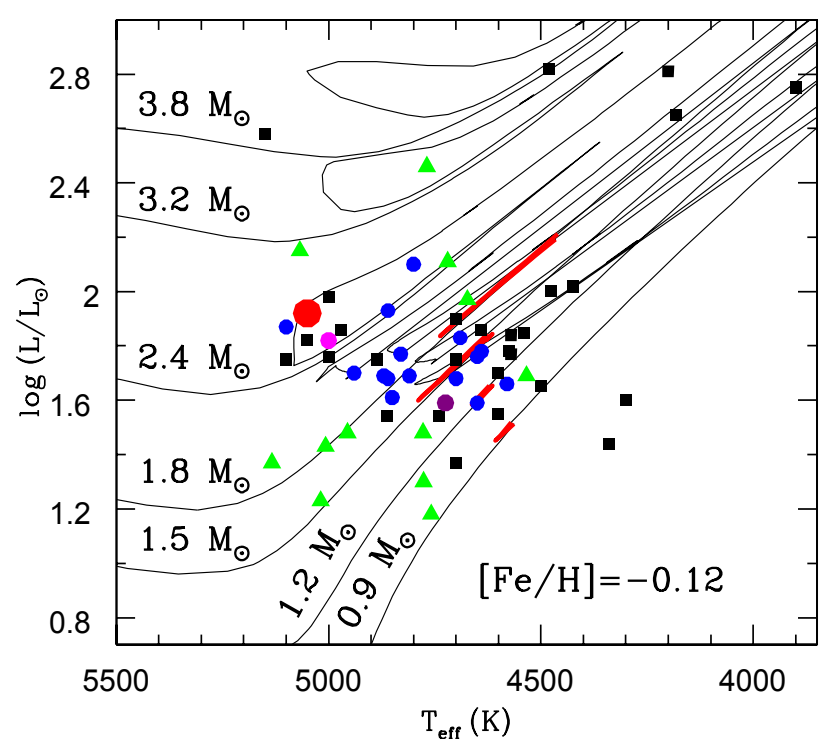

Fig. 3. Position of HD 16771 (red) in the $\log \left(L / L_{\odot}\right)$ versus $T_{\text {eff }}$ plane along with other Li-rich giants (see text). The location of the LFB is indicated by red stripes. HD 16771 with $T_{\text {eff }}=5050 \mathrm{~K}$ is clearly on the high temperature side of the bump found for low-mass stars where the possibility of extra-mixing is proposed (Charbonnel \& Balachandran 2000).

$\log g=2.6-3.1$ and ${ }^{12} \mathrm{C} /{ }^{13} \mathrm{C} \leq 15$. The study of Brown et al. (1989) has included a clump-giant HD 120602 with $T_{\text {eff }}=$ 5000, $\log g=3.00$ whose ${ }^{12} \mathrm{C} /{ }^{13} \mathrm{C}$ has been measured at 16 (Berdyugina \& Savanov 1994). HD 16771 with ${ }^{12} \mathrm{C} /{ }^{13} \mathrm{C}=$ $12 \pm 2$ is another such example whose $\mathrm{Li}$ enrichment is not linked to the episode of extra-mixing near the LFB or He-core flash as suggested previously for stars of mass $M<2.2 M_{\odot}$ (Charbonnel \& Balachandran 2000; Kumar et al. 2011). Here, we avoid plotting the sample of Martell \& Shetrone (2013) in Fig. 3, as they have no measured parallaxes or $V$ magnitudes.

However, the observed low values of $\mathrm{C} / \mathrm{N} \sim 0.7$ and ${ }^{12} \mathrm{C} /{ }^{13} \mathrm{C} \sim 12$, although far from the predictions of conventional mixing (Charbonnel et al. 1998), clearly show that HD 16771 has experienced non-canonical mixing and thus the possibility of preserving initial main-sequence lithium content and external pollution from a companion is excluded. Moreover, the external pollution from an evolved Li-rcih AGB companion is associated with the enhancements in the $s$-process elements, characteristics not carried by HD 16771. Together five epochs of
RV observations collected from Mermilliod et al. (2008) show no sign of binarity.

Thus, the lithium overabundance in HD 16771 requires an alternative to models of extra-mixing (see Carlberg et al. 2012 and references therein) that invoke destruction of $\mu$-barrier in solar metallicity stars with degenerate He cores $\left(M<2.2 M_{\odot}\right)$ that have evolved through both the LFB and He-core flash stages. However, for high mass stars $\left(M>2.2 M_{\odot}\right)$ with nondegenerate He cores, $\mathrm{Li}$ evolution ceases once the star completes first dredge-up.

If fresh ${ }^{7} \mathrm{Be}$ is synthesized via Cameron-Fowler mechanism in the interior of a star like HD 16771, the mixing should be fast enough to carry ${ }^{7} \mathrm{Be}$ (and ${ }^{7} \mathrm{Li}$ ) to cool layers where it decays to ${ }^{7}$ Li. Indeed, Denissenkov (2012) proposed a stirring mechanism in the RGB phase of low-mass stars with fast internal rotation which leads to a temperature gradient between the hydrogenburning shell and the envelope that permits enhanced mixing across the radiative zone. As a consequence, a low-mass star make extended zigzags toward the position of the red-clump star, before resuming its ascent along the RGB. As the theoretical models do not predict $\mathrm{Li}$ production during the He-core flash, Denissenkov (2012) proposed that the red-clump stars in the study of Kumar et al. (2011) are LFB low-mass stars which had made their excursion in the HR-diagram towards luminosities compatible with the expected location of red-clump stars. However, our star has a very low probability to encounter such scenario and sits on the high temperature side and far away from the LFB found for low mass stars (Fig. 3).

Several other sources of enhanced extra-mixing have been suggested to explain the presence of Li-rich giants all along the RGB. Among those are Denissenkov \& Weiss (2000) and Denissenkov \& Herwig (2004) models of activation of ${ }^{7} \mathrm{Be}-$ transport mechanism followed by the engulfing of a giant planet (Alexander 1967). The engulfing of close-in planets might cause shear instabilities at the base of the convective envelope that could turn-on the dynamo action (Siess \& Livio 1999). The associated thermohaline (Charbonnel \& Zahn 2007) and magnetothermohaline mixing (Denissenkov et al. 2009) could circulate the material fast enough to replenish $\mathrm{Li}$ in the stellar envelope that also lowers the ${ }^{12} \mathrm{C} /{ }^{13} \mathrm{C}$ ratio from its first dredge-up value.

Exoplanets observations show deficiency of close-in planets around evolved stars compared to their main sequence counterparts, for which planet engulfment is likely a plausible explanation (Villaver et al. 2014). In principle, HD 16771 has evolved enough to engulf a putative close-in planet, as in known hot Jupiter systems, for which the semi major axis is usually less than 0.05 AU (Adamów et al. 2015). On the account of positive detection of Li excess in HD 16771 at the red-clump phase, we speculate that the engulfing of planet would have occurred during RGB stage, where the giant's outer envelope expands and reaches to its maximum stellar radius at the RGB tip. And for stars with $M=2.4 M_{\odot}$ the critical initial semi-major axis for engulfment is $\sim 0.12-0.18 \mathrm{AU}$ and for giant stars in the age range 620-650 Myr (Delgado Mena et al. 2016). Therefore, HD 16771 with an age of $760 \pm 130 \mathrm{Myr}$ would have undergone extra-mixing triggered by the engulfment of planet(s) probably $100 \mathrm{Myr}$ ago and yet the observed Li overabundance suggest that the elapsed time is insufficient for Li dilution. Also the rapid evolution from the RGB to red-clump phase and the shallow convective envelope of the He-core burning stars could aid in preserving $\mathrm{Li}$ as the envelope material hardly circulates to the hot internal layers where Li is promptly destroyed (Delgado Mena et al. 2016). 
Siess \& Livio (1999) have suggested that engulfment of substellar objects not only raise the star's ${ }^{7} \mathrm{Li}$ abundance but could also trigger a prompt mass-loss, spin-up of the star, and the possible generation of magnetic fields, depending on the masses of both star and planet and on the planet's orbital parameters. The slow rotation of $2.8 \mathrm{~km} \mathrm{~s}^{-1}$, typical for an evolved giant, suggest that HD 16771 could have undergone efficient rotational braking in this time frame and carried away most of the envelope's angular momentum in a mass-loss event. Others who have considered planet accretion as the solution to $\mathrm{Li}$ enhancement in some giant stars include Adamów et al. (2012), Carlberg et al. (2012) and Delgado Mena et al. (2016). A major fraction of Li-rich giants with planets in those studies present $v \sin i<4 \mathrm{~km} \mathrm{~s}^{-1}$, and our giant would be one such candidate showing a slow rotational velocity. These results, as previously noted by Fekel \& Balachandran (1993), weaken the claim that planet ingestion, high lithium abundance and rapid rotation are related inextricably, as the timescales for $\mathrm{Li}$ enrichment are not necessarily the same as the timescales for rotational spin-up and spin-down, or for increased or decreased $\mathrm{Ca}$ II $\mathrm{H}$ and $\mathrm{K}$ emission.

A thorough investigation of IR fluxes extracted from 2MASS (Cutri et al. 2003) and WISE (Wright et al. 2010) reveals no sign of IR excess at short wavelengths (i.e., $J, K$, and WISE[3.4, 4.6, 12 and $22 \mu \mathrm{m}]$ ). Using the test given by de La Reza et al. (1996), which makes use of the [60-25] and [25-12] IRAS data, we confirm that HD 16771, in common with BD+48 740 (Adamów et al. 2012), shows IR excess at $60 \mu \mathrm{m}$, suggesting that the engulfment happened some time ago allowing the shell time to expand and cools down. Supporting this assertion are the lack of observations of asymmetric $\mathrm{H} \alpha$ and irregular $\mathrm{Na} \mathrm{D}$ lines in our spectra, which are expected as a result of very recent event of intense mass-loss. Computations show that the dust shell expansion to produce excess emission at $60 \mu \mathrm{m}$ will take about $10^{4} \mathrm{yr}$ (de La Reza et al. 1996), which is consistent with the proposal that mass-loss would have carried away most of the envelope's angular momentum and, eventually, slow down HD 16771 to the observed value of $v \sin i=2.8 \mathrm{~km} \mathrm{~s}^{-1}$ in the red-clump phase. Along these lines we speculate that HD 16771 is in the cool extended dust shell and high lithium abundance phase of Siess \& Livio's (1999) scenario.

Another signature of planet engulfment is the detection of ${ }^{9}$ Be enrichment in stars. A search for beryllium enhancements in a limited sample of Li-rich and Li-normal giants with $T_{\text {eff }}=$ $3900-4800 \mathrm{~K}$ and masses in the range $1.4-3.2 M_{\odot}$ provides no detection of the ${ }^{9} \mathrm{Be}$ line at $3131 \AA$ (Melo et al. 2005). Although, the Be II line at $3131 \AA$ falls outside our spectral range to measure its abundance, its usefulness for deriving Be abundances decreases with temperature below $5400 \mathrm{~K}$ to a point where it will be useless (Santos et al. 2004). The source of difficulty arises from the severe blending of Be line with other atomic lines and their dominance at low temperatures around $3131 \AA$, and the missing near-UV opacity which is not needed at the solar effective temperature (see Santos et al. 2004, and references therein). Therefore, the Be abundance determinations requires further scrutiny.

We note here that engulfing a putative close-in planet(s) with an equivalent mass of 30 Jupiters (using Eq. (2) in Siess \& Livio 1999), whose atmosphere was hydrogen-poor (say by about $1.5 \mathrm{dex}$ ) due to the evaporation of hydrogen atoms by the sustainable radiation pressure from the host star (Alexander 1967; Vidal-Madjar et al. 2003), into a reasonable convective envelope of $80 \%$ the mass of the red giant (Brown et al. 1989) would raise the lithium abundance of HD 16771 to 2.8 dex and the beryllium abundance by 1.0 dex without affecting appreciably the abundances of other elements. The above calculations are made for a giant star whose convective envelope is void of both the lithium and beryllium abundances. However, the true enhancement in the beryllium abundance would be much smaller (about 0.4 dex if we assume a beryllium abundance of 0.8 dex on the RGB) and probably undetectable because of severe blending of Be line with other atomic lines and the difficulty in continuum placement in those crowded regions of the spectra. Then a prompt depletion of ${ }^{6} \mathrm{Li}$ and a progressive dilution of ${ }^{7} \mathrm{Li}$ would naturally explain $\mathrm{Li}$ overabundance and IR excess in HD 16771 without any need for Cameron-Fowler mechanism. Therefore, the absence/presence of ${ }^{9} \mathrm{Be}$ overabundance in HD 16771 would not necessarily contradict the engulfment scenario, as the close-in planets are fated to undergo regular radiational harassment from their host stars which would eventually evaporate hydrogen much faster than other elements (Vidal-Madjar et al. 2003; Bourrier et al. 2014).

The puzzling overabundance of lithium in HD 16771 makes it an oustanding prospect for future studies to set strong constraints on the role of planet engulfment and the episode of $\mathrm{Li}$ synthesis for stars that neither experience LFB nor the He-core flash, and on the timescales for Li dilution, rotational spin-up and spin-down of stars.

Acknowledgements. We are grateful to the McDonald Observatory's Time Allocation Committee for granting us observing time for this project. D.L.L. wishes to thank the Robert A. Welch Foundation of Houston, Texas for support through grant F-634. We are grateful to the anonymous referee for a very careful and constructive report that led to improvements of the Paper. This research has made use of the WEBDA database, operated at the Department of Theoretical Physics and Astrophysics of the Masaryk University and the NASA ADS, USA. This research has made use of the SIMBAD database and Aladin sky atlas, operated at CDS, Strasbourg, France. This publication makes use of data products from the Two Micron All Sky Survey, which is a joint project of the University of Massachusetts and the Infrared Processing and Analysis Center/California Institute of Technology, funded by the National Aeronautics and Space Administration (NASA) and the National Science Foundation (NSF).

\section{References}

Adamów, M., Niedzielski, A., Villaver, E., Nowak, G., \& Wolszczan, A. 2012, ApJ, 754, L15

Adamów, M., Niedzielski, A., Villaver, E., Wolszczan, A., \& Nowak, G. 2014, A\&A, 569, A55

Adamów, M., Niedzielski, A., Villaver, E., et al. 2015, A\&A, 581, A94

Afşar, M., Sneden, C., \& For, B.-Q. 2012, AJ, 144, 20

Alexander, J. B. 1967, The Observatory, 87, 238

Alonso, A., Arribas, S., \& Martínez-Roger, C. 1999, A\&AS, 140, 261

Berdyugina, S. V., \& Savanov, I. S. 1994, Astron. Lett., 20, 639

Bourrier, V., Lecavelier des Etangs, A., \& Vidal-Madjar, A. 2014, A\&A, 565, A105

Bressan, A., Marigo, P., Girardi, L., et al. 2012, MNRAS, 427, 127

Brown, J. A., Sneden, C., Lambert, D. L., \& Dutchover, Jr., E. 1989, ApJS, 71, 293

Cameron, A. G. W., \& Fowler, W. A. 1971, ApJ, 164, 111

Carlberg, J. K., Cunha, K., Smith, V. V., \& Majewski, S. R. 2012, ApJ, 757, 109

Castelli, F., \& Kurucz, R. L. 2004, Proc. IAU Symp., 210, A20

Charbonnel, C., \& Balachandran, S. C. 2000, A\&A, 359, 563

Charbonnel, C., \& Lagarde, N. 2010, A\&A, 522, A10

Charbonnel, C., \& Zahn, J.-P. 2007, A\&A, 467, 15

Charbonnel, C., Brown, J. A., \& Wallerstein, G. 1998, A\&A, 332, 204

Cutri, R. M., Skrutskie, M. F., van Dyk, S., et al. 2003, The IRSA 2MASS

All-Sky Point Source Catalog (Pasadena, CA: IPAC/California Institute of Technology)

da Silva, L., Girardi, L., Pasquini, L., et al. 2006, A\&A, 458, 609

de La Reza, R., Drake, N. A., \& da Silva, L. 1996, ApJ, 456, L115

Delgado Mena, E., Tsantaki, M., Sousa, S. G., et al. 2016, A\&A, 587, A66

Denissenkov, P. A. 2012, ApJ, 753, L3

Denissenkov, P. A., \& Herwig, F. 2004, ApJ, 612, 1081

Denissenkov, P. A., \& Weiss, A. 2000, A\&A, 358, 49

Denissenkov, P. A., Pinsonneault, M., \& MacGregor, K. B. 2009, ApJ, 696, 1823

Fekel, F. C., \& Balachandran, S. 1993, ApJ, 403, 708 
Ghezzi, L., Cunha, K., Smith, V. V., et al. 2009, ApJ, 698, 451

Iben, I., 1967a, ApJ, 147, 624

Iben, I., 1967b, ApJ, 147, 650

Jofré, E., Petrucci, R., García, L., \& Gómez, M. 2015, A\&A, 584, L3

Jones, B. F., \& Prosser, C. F. 1996, AJ, 111, 1193

Kumar, Y. B., Reddy, B. E., \& Lambert, D. L. 2011, ApJ, 730, L12

Lambert, D. L., \& Dearborn, D. S. 1972, Mem. Soc. Roy. Sci. Liège, 6th Series, 3, 147

Lind, K., Asplund, M., \& Barklem, P. S. 2009, A\&A, 503, 541

Martell, S. L., \& Shetrone, M. D. 2013, MNRAS, 430, 611

Masseron, T., Plez, B., Van Eck, S., et al. 2014, A\&A, 571, A47

Melo, C. H. F., de Laverny, P., Santos, N. C., et al. 2005, A\&A, 439, 227

Mermilliod, J.-C., \& Mayor, M., \& Urdy, S. 2008, A\&A, 485, 303

Monaco, L., Villanova, S., Moni Bidin, C., et al. 2011, A\&A, 529, A90

Nicolet, B. 1978, A\&AS, 34, 1

Ram, R. S., Brooke, J. S. A., Bernath, P. F., Sneden, C., \& Lucatello, S. 2014 ApJS, 211, 5

Reddy, A. B. S., Giridhar, S., \& Lambert, D. L. 2012, MNRAS, 419, 1350
Reddy, A. B. S., Giridhar, S., \& Lambert, D. L. 2013, MNRAS, 431, 3338 Reddy, A. B. S., Giridhar, S., \& Lambert, D. L. 2015, MNRAS, 450, 4301

Sackmann, I.-J., \& Boothroyd, A. I. 1992, ApJ, 392, L71

Sackmann, I.-J., \& Boothroyd, A. I. 1999, ApJ, 510, 217

Santos, N. C., Israelian, G., Randich, S., et al. 2004, A\&A, 425, 1013

Siess, L., \& Livio, M. 1999, MNRAS, 308, 1133

Silva Aguirre, V., Ruchti, G. R., Hekker, S., et al. 2014, ApJ, 784, L16

Sneden, C. 1973, Ph.D. Thesis, Univ. of Texas, Austin

Sneden, C., Pilachowski, C. A., \& Vandenberg, D. A. 1986, ApJ, 311, 826

Sneden, C., Lucatello, S., Ram, R. S., Brooke, J. S. A., \& Bernath, P. 2014, ApJS, 214,26

Tull, R. G., MacQueen, P. J., Sneden, C., \& Lambert, D. L. 1995, PASP, 107, 251

Vidal-Madjar, A., Lecavelier des Etangs, A., Désert, J.-M., et al. 2003, Nature, 422,143

Villaver, E., Livio, M., Mustill, A. J., \& Siess, L. 2014, ApJ, 794, 3

Wright, E. L., Eisenhardt, P. R. M., Mainzer, A. K., et al. 2010, AJ, 140, 1868 